\title{
AUTOPERCEPÇÃO VOCAL DE CORISTAS PROFISSIONAIS
}

\author{
Vocal self-perception of professional singers
}

\author{
Fernanda Salvatico de Aquino(1), Lídia Cristina da Silva Teles(2)
}

\begin{abstract}
RESUMO
Objetivo: identificar o nível de autopercepção vocal de cantores de um coral profissional. Método: participaram 44 coristas, com idades entre 20 e 75 anos ( $\bar{X} 51,5 \pm 14,36$ ), de ambos os sexos. Estes responderam a um questionário com 30 questões objetivas sobre autopercepção vocal e queixas da voz cantada e falada; experiência com o canto e hábitos relacionados ao bem estar vocal. Resultados: todos os coristas auto definiram suas vozes com características positivas, não apresentando diferença estatística entre voz falada e cantada. Os coristas identificaram também características vocais negativas para voz falada e cantada, sendo que foi encontrada diferença estatística. O índice de queixas vocais foi de $31 \%$ para voz falada e $25 \%$ para voz cantada, sem apresentar diferença estatística. $43 \%$ dos participantes realizaram aulas de canto, com tempo médio 1,95 anos ( $\pm 2,29$ anos) e o tempo de participação em corais variou de seis meses a 66 anos, com tempo médio de 17,74 anos ( $\pm 18,45$ anos). Quanto aos hábitos relacionados ao bem estar vocal, o índice médio de ingestão diária de água foi de 1,7 litros ( \pm 0,92 litros); $50 \%$ dos coristas fazem ingestão de álcool com frequência; o índice numérico de tabagistas foi de $4,5 \%$ e $22 \%$ dos coristas são ex-tabagistas. Não foram encontradas correlações estatísticas entre tabagismo, etilismo e experiência no canto com queixas vocais. Conclusão: os coristas avaliados apresentaram um nível elevado de autopercepção de seus aspectos vocais, podendo sugerir que o canto coral desempenha um papel importante na promoção do bem estar vocal.
\end{abstract}

DESCRITORES: Voz; Envelhecimento; Hábitos; Autoimagem

\section{INTRODUÇÃO}

O canto está presente desde a antiguidade em diversas culturas, relacionado à magia, saúde, cerimônias religiosas, festas e guerras. É uma das formas mais belas de arte, que expressa o que as palavras por si podem muitas vezes não conseguir. Contudo, o canto não se trata apenas de uma forma de expressão, mas de uma socialização que modifica valores. A maneira singular de o artista olhar o mundo influi nos julgamentos realizados sobre si próprio. O prazer que esse trabalho proporciona motiva o cantor ${ }^{1}$.

(1) Fonoaudióloga; Mestranda do Programa de Pós-Graduação em Fonoaudiologia da Pontifícia Universidade Católica de São Paulo - PUC-SP, São Paulo, SP, Brasil.

(2) Fonoaudióloga; Professora Doutora do Departamento de Fonoaudiologia da Faculdade de Odontologia de Bauru da Universidade de São Paulo - FOB/USP, Bauru, SP, Brasil.

Conflito de interesses: inexistente
A produção do canto utiliza as mesmas estruturas da voz falada, porém com ajustes necessários à interpretação musical. A respiração no canto é treinada, sendo mais rápida que para a fala, com ciclos predeterminados de acordo com as frases musicais. No canto usa-se um maior volume de ar, que deve ser controlado durante sua saída, com movimentação das paredes do tórax. Os ciclos vibratórios das pregas vocais possuem um cociente de fechamento maior que o de abertura, que proporciona ao cantor maior duração de tempo e com um som mais rico em harmônicos. Durante o canto a laringe tende a permanecer em posição mais baixa e estabilizada, mesmo em frequências mais agudas. A ressonância do cantor é geralmente alta, com concentração na parte superior do trato vocal. O cantor possui um melhor controle do fluxo aéreo expiratório que proporciona riqueza na variação da loudness e permite maior projeção da voz. Outro aspecto importante para o cantor é ter grande extensão de frequência vocal, geralmente cerca de duas oitavas e meia, maior que a utilizada 
para a fala. Todos estes fatores aliados aos treinos vocais dos cantores contribuem para que a qualidade vocal na voz cantada seja mais estável. Portanto, pode-se considerar o canto um excelente treinamento vocal.

Há autores que aludem que alterações vocais podem ser combatidas por meio do canto, que influencia diretamente na qualidade da $\mathrm{voz}^{2,3}$. Segundo Fuchs et al. (2009) ${ }^{4}$, o canto auxilia ainda na autopercepção vocal e pode ser um dos fatores que leva o indivíduo a apresentar maiores cuidados, cometendo menos abusos, não gritando e usando a voz de maneira mais sofisticada em relação aos indivíduos que não cantam. Dessa forma, a experiência do canto influencia diretamente na performance vocal, bem como na autopercepção da voz pelo indivíduo.

Para Bicalho et al. $(2010)^{5}$, profissionais que trabalham com a voz podem atribuir valores e importâncias diferentes na autopercepção de sua qualidade vocal.

A autopercepção vocal tem apresentado grande valor na prática clínica nos dias de hoje, por captar a perspectiva do indivíduo em relação ao impacto que acredita que sua voz produza nos ouvintes. Kasama e Brasolotto $(2007)^{6}$, sugeriram que a autopercepção vocal está intimamente ligada à qualidade de vida, podendo ser usada como instrumento na detecção de problemas relacionados à comunicação. Entretanto, não há relatos na literatura sobre a autopercepção vocal de cantores, profissionais estes que apresentam grande demanda de uso da voz e alta exigência quanto à qualidade vocal.

O objetivo desse trabalho foi identificar o nível de autopercepção dos aspectos vocais de cantores de um coral profissional.

\section{MÉTODO}

Esse estudo foi aprovado pelo Comitê de Ética em Pesquisa da Faculdade de Odontologia de Bauru-USP sob o Protocolo de Pesquisa no 109/204. Foram respeitados todos os princípios éticos que versam a resolução 196/96 e 257/97 sobre ética em pesquisa com seres humanos e as orientações do Comitê de Ética em Pesquisa da Faculdade de Odontologia de Bauru-USP.

Trata-se de um estudo transversal e descritivo, que analisou os dados obtidos do questionário aplicado no projeto de pesquisa "Características da fonetografia em coristas de diferentes classificações vocais"'.

Foram analisados os dados obtidos do questionário aplicado no projeto de pesquisa a que se submeteram 44 indivíduos, integrantes de um coral profissional da cidade de Bauru/SP, sendo 16 homens e 28 mulheres, com idades entre 20 e 75 anos (X 51,5 anos $\pm 14,36$ anos).

Para a realização do estudo, os coristas participantes responderam a um questionário (Figura 1) contendo 30 questões objetivas, que foi aplicado de forma verbal e suas respostas anotadas pelas pesquisadoras a fim de levantar informações referentes à:

I) Voz cantada: autopercepção da voz (cinco características positivas e cinco negativas observadas na voz); queixa da voz cantada (se há desconforto vocal após o cantar) e experiência no canto (se realizou aulas de canto, durante quanto tempo e há quanto tempo participa em corais).

II) Voz falada: autopercepção da voz listando quais as características observadas na voz sendo cinco positivas (bonita, agradável, clara, forte e suave) e cinco negativas (feia, ardida, abafada, fraca e áspera) e queixas vocais (rouquidão, afonia, dor).

III) Hábitos relacionados à saúde vocal: quantidade de ingestão de água ao longo do dia; uso frequente de álcool e tabagismo.

A autopercepção da voz positiva ou negativa foi classificada em baixa, média ou alta de acordo com o número de características citadas pelos coristas, em que até duas características citadas é considerado um nível baixo, três um nível médio e de 4 a 5, um nível alto.

A análise estatística dos dados obtidos se deu por meio do Teste de Hipótese que adotou nível de significância de $95 \%$. Foram comparados os intervalos de confiança dos escores de voz cantada e voz falada em seus diferentes níveis adotados, tanto para aspectos positivos e negativos, quanto para o índice de queixas.

Com a intenção de obter a correlação dos demais índices foi utilizada a Estatística de Regressão, a partir do teste R-Quadrado que adota nível de correlação igual a 1,0. Por meio dele pode-se correlacionar os hábitos vocais negativos, o tempo de experiência em corais e a realização de aulas de canto com queixas vocais na voz falada. 
Nome:

Sexo:

Idade:

Tempo de participação em corais:

I. Em relação à sua voz CANTADA, você a considera:
1. Bonita
$S() \quad N()$
2. Agradável
$S($ ) $\quad N()$
3. Clara
$S() \quad N()$
4. Forte
$S() \quad N()$
5. Suave
$S() \quad N()$
6. Feia
$S() \quad N()$
7. Ardida
$S() \quad N()$
8. Abafada
$S() \quad N()$
9. Fraca
$S() \quad N()$
10. Áspera
$S() \quad N()$

11. Você sente algum desconforto vocal após cantar?

12. Você já realizou ou realiza aula de canto?

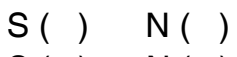

13. Se sim, durante quanto tempo?

II. Em relação à sua voz FALADA, você a considera:
1. Bonita
$S() \quad N()$
2. Agradável
$S($ ) $N()$
3. Clara
$S() \quad N()$
4. Forte
$S() \quad N()$
5. Suave
$S() \quad N()$
6. Feia
$S() \quad N()$
7. Ardida
$S() \quad N()$
8. Abafada
$S() \quad N()$
9. Fraca
$S() \quad N()$
10. Áspera
$S() \quad N()$

11. Você sente sua voz rouca? $\quad S($ ) $\quad N($ )

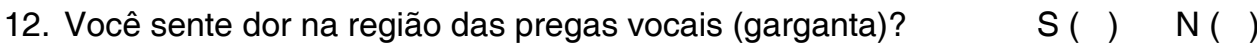

13. Você apresenta afonia com frequência (perde a voz com frequencia)? $S($ ) N ( )

III. Hábitos relacionados à saúde vocal:

1. Você fuma? $\mathrm{S}(\mathrm{N}) \mathrm{N}(\mathrm{)}$

2. Já fumou? $\mathrm{S}(\mathrm{)} \quad \mathrm{N}(\mathrm{)})$

3. Ingere bebida alcoólica com frequência (pelo menos uma vez na semana)?S ( ) N ( )

4. Você ingere que quantidade de água durante o dia?

Figura 1 - Questionário 


\section{RESULTADOS}

Todos os coristas apresentaram autopercepção vocal positiva tanto para a voz cantada como falada, sendo que $62 \%$ deles referiram quatro ou mais características positivas em suas vozes, classificada portanto, como alta percepção positiva da voz (Figura 2).

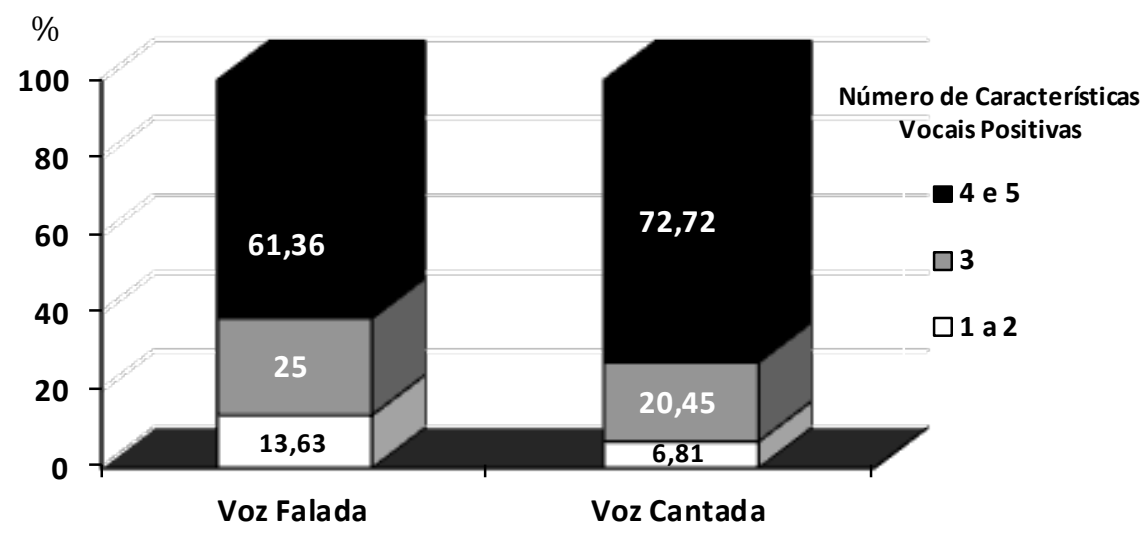

Figura 2 - Percentagens do número de características vocais positivas da voz falada e cantada referidas pelos coristas deste estudo

A análise estatística constatou que os índices de autopercepção não apresentam diferença entre voz falada e voz cantada (Tabela 1).

A autopercepção vocal negativa foi encontrada em $36 \%$ dos coristas para a voz falada e em $30 \%$ para a voz cantada, sendo que a maioria destes ( $32 \%$ voz falada e $25 \%$ voz cantada) referiu apenas uma ou duas características negativas, sendo baixa a classificação da autopercepção vocal negativa (Figura 3).

Tabela 1 - Intervalos de confiança dos escores obtidos da autopercepção positiva de voz falada e voz cantada

\begin{tabular}{ccccccc}
\hline & \multicolumn{2}{c}{ Nível Baixo } & \multicolumn{2}{c}{ Nível Médio } & \multicolumn{2}{c}{ Nível Alto } \\
\cline { 2 - 6 } & $\begin{array}{c}\text { Limite } \\
\text { Inferior }\end{array}$ & $\begin{array}{c}\text { Limite } \\
\text { Superior }\end{array}$ & $\begin{array}{c}\text { Limite } \\
\text { Inferior }\end{array}$ & $\begin{array}{c}\text { Limite } \\
\text { Superior }\end{array}$ & $\begin{array}{c}\text { Limite } \\
\text { Inferior }\end{array}$ & $\begin{array}{c}\text { Limite } \\
\text { Superior }\end{array}$ \\
\cline { 2 - 7 } Voz Falada & $54,93 \%$ & $67,79 \%$ & $19,28 \%$ & $30,72 \%$ & $9,10 \%$ & $18,16 \%$ \\
Voz Cantada & $66,83 \%$ & $78,61 \%$ & $15,12 \%$ & $25,78 \%$ & $3,48 \%$ & $10,14 \%$ \\
\hline Resultado & Estatisticamente Iguais & Estatisticamente Iguais & Estatisticamente Iguais \\
\hline
\end{tabular}

Teste de Hipóteses: $p=95 \%$

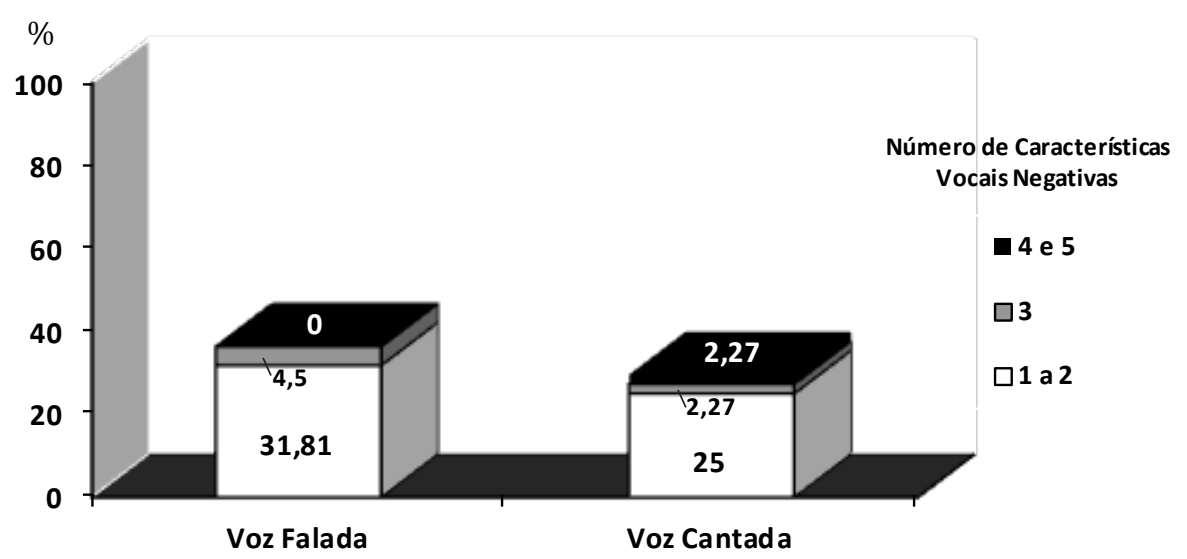

Figura 3 - Percentagens do número de características vocais negativas da voz falada e cantada referidas pelos coristas deste estudo 
A análise estatística constatou que os níveis baixo e médio de autopercepção vocal negativa não apresentaram diferença entre voz falada e voz canta. Entretanto, o nível alto apresentou diferença estatisticamente significante (Tabela 2).

As alterações vocais na voz falada foram relatadas por $31 \%$ dos participantes que referiram presença de rouquidão, afonia e/ou dor. $\mathrm{Na}$ voz cantada, $25 \%$ dos indivíduos relataram presença de desconforto vocal após o canto. Por meio da análise estatística foi possível constatar que não existe diferença entre as queixas referidas na voz falada e na voz cantada. Os intervalos de confiança foram de $19,28 \%$ e $30,72 \%$ para voz cantada e $25,66 \%$ e $37,96 \%$ para voz falada.

Ao serem questionados, os participantes não referiram presença de alterações laríngeas, constatada em exame otorrinolaringológico.
A experiência no canto foi analisada considerando a participação em aulas de canto e tempo de atuação em corais. Dezenove (43\%) dos 44 coristas frequentam ou frequentaram aulas de canto durante um tempo que variou de três meses a quatro anos ( $\overline{\mathrm{X}}$ 1,95anos $\pm 2,29$ anos). O tempo de participação em corais variou de 6 meses a 66 anos, com tempo médio de 17,74 anos ( $\pm 18,45$ anos).

Dos hábitos relacionados à saúde vocal observou-se que os coristas ingerem em média 1,7 litros de água ( $\pm 0,92$ litros). Quantos aos hábitos negativos $50 \%$ referiram ingerir bebidas alcoólicas com frequência (uma vez por semana ou mais); $4,5 \%$ são tabagistas e $22,7 \%$ ex-tabagistas.

Quanto a correlação entre hábitos vocais negativos, tempo de experiência em corais e realização de aulas de canto com queixas vocais na voz falada, os índices encontrados não foram estatisticamente significantes (Tabela 3).

Tabela 2 - Intervalos de confiança dos escores obtidos da autopercepção negativa de voz falada e voz cantada

\begin{tabular}{ccccccc}
\hline & \multicolumn{2}{c}{ Nível Baixo } & \multicolumn{2}{c}{ Nível Médio } & \multicolumn{2}{c}{ Nível Alto } \\
\cline { 2 - 7 } & Limite & Limite & Limite & Limite & Limite & Limite \\
& Inferior & Superior & Inferior & Superior & Inferior & Inferior \\
\cline { 2 - 7 } Voz Falada & $25,66 \%$ & $37,96 \%$ & $1,76 \%$ & $7,24 \%$ & $\mathbf{0 , 0 0 \%}$ & $\mathbf{0 , 0 0 \%}$ \\
Voz Cantada & $19,28 \%$ & $30,72 \%$ & $0,30 \%$ & $4,24 \%$ & $\mathbf{0 , 3 0 \%}$ & $\mathbf{4 , 2 4 \%}$ \\
\hline \multirow{2}{*}{ Resultado } & \multirow{2}{*}{ Estatisticamente Iguais } & \multicolumn{2}{c}{ Estatisticamente Iguais } & \multicolumn{2}{c}{$\begin{array}{c}\text { Estatisticamente } \\
\text { Diferentes }\end{array}$} \\
\hline
\end{tabular}

Teste de Hipóteses: $p=95 \%$

Tabela 3 - Correlação entre hábitos vocais negativos, tempo de experiência em corais e realização de aulas de canto com queixas vocais na voz falada

\begin{tabular}{ccc}
\hline & Queixas Vocais na Voz Falada & Resultado \\
\hline Tabagismo e Etilismo & 0,009 & Não Significante \\
Tempo de Experiência em Corais & 0,005 & Não Significante \\
Realização de Aulas de Canto & 0,004 & Não Significante \\
\hline
\end{tabular}

R-Quadrado: $p=1,0$

\section{DISCUSSÃO}

O canto coral é uma prática humana, que além de propiciar a vivência em grupo, envolvendo todos seus aspectos sociais, proporciona também uma melhor autopercepção, desenvolve a autoestima do corista e valoriza sua individualidade ${ }^{1,8}$.

No presente trabalho pode-se constatar que a maior parte dos integrantes do coral avaliado possui autopercepção vocal positiva elevada e autopercepção negativa reduzida, tanto para a voz falada quanto cantada. Tal fato pode ser atribuído ao melhor desempenho vocal que pressupõe-se que os cantores apresentem. Este dado vai ao encontro do estudo de Fuchs $(2009)^{4}$, que concluíram que a experiência no canto influencia diretamente na performance vocal, bem como na autopercepção da voz pelo indivíduo.

Embora não apresente correlação estatística com as queixas vocais, um fator que pode 
influenciar a autopercepção vocal é o tempo de experiência com o canto. Para Amato $(2007)^{8}$, a vivência em grupo que o canto coral proporciona estimula a automotivação do corista, que apresenta grande crescimento pessoal, estabelecendo uma rede sociocultural a partir da valorização de sua individualidade. Além disso, essa experiência faz com que o corista tenha a forma de olhar o mundo transformada pela prática social e enriquecedora que é o canto coral ${ }^{1}$.

Quanto à participação em aulas de canto, foi possível observar que apenas $43 \%$ dos coristas em questão buscaram aperfeiçoamento em técnicas vocais por meio de aulas de canto. Mesmo não apresentando correlação estatística com as queixas, tal fato pode ser um alerta para os profissionais que cuidam do bem estar vocal dos cantores, que devem se preocupar em garantir informação e orientação a esses indivíduos.

Outro aspecto a ser apontado é que, na comparação estatística entre voz falada e voz cantada, apenas o índice do nível alto de autopercepção negativa, conforme apontam os dados da Tabela 2 , apresentou diferença, sendo maior para voz cantada. A percepção de características negativas da voz, principalmente no que se refere à voz cantada, sugere que os coristas possuem uma sensibilidade refinada na identificação de pequenas alterações, se mostrando críticos quanto à qualidade de sua voz devido à demanda que apresentam.

Esse achado também pode estar indiretamente relacionado aos ajustes laríngeos, respiratórios e do trato vocal que o corista utiliza durante o canto, que lhe proporciona um melhor desempenho vocal e também maior exigência. A esse respeito Bicalho et al. $(2010)^{5}$ referiram que os profissionais que trabalham com a voz podem atribuir valores e importâncias diferentes na sua autopercepção, sendo mais exigentes em alguns aspectos.

Esse dado corrobora o fato de os cantores apresentarem queixas vocais, ainda que não exista diferença estatística entre voz falada e voz cantada.

Observou-se, especialmente, não haver queixa ou relato de alterações laríngeas entre as mulheres no período da menopausa. No estudo de Machado et al. $(2005)^{9}$, os autores sugeriram que mulheres identificam essas modificações vocais que ocorrem em decorrência da menopausa. No entanto, deve-se levar em consideração o fato de não haver avaliação laríngea que confirme os relatos obtidos. A literatura refere aumento de queixas e de alterações vocais em mulheres na menopausa, bem como decorrentes do envelhecimento, em idosos não cantores ${ }^{9-11}$. Pode-se inferir que o canto, para essas coristas, tenha fortalecido as estruturas da laringe e prevenido ou minimizado as alterações vocais decorrentes das mudanças hormonais ou do envelhecimento. Para Brown et al. (1990) ${ }^{3}$, o canto pode influenciar diretamente na qualidade vocal da terceira idade. Em seu estudo, a voz dos idosos cantores e dos adultos jovens apresentou um melhor desempenho comparativamente à de idosos não cantores. Hazlett e Ball (1996) ${ }^{2}$, relataram que o tempo de experiência no canto e os efeitos do treinamento vocal de cantores podem combater mudanças vocais que ocorrem devido ao envelhecimento.

Quanto aos hábitos relacionados ao bem estar vocal, a ingestão média de 1,7 litros de água que os coristas fazem ao longo dia demonstrou que esses estão cientes da necessidade de hidratação. A literatura indica que esse é um aspecto positivo, uma vez que um bom nível de hidratação é importante para todos os seres vivos, e principalmente para o indivíduo que faz uso profissional da voz, por prevenir a fadiga vocal ${ }^{12}$.

Com relação aos hábitos negativos, esses também não apresentaram correlação estatística com as queixas vocais. Entretanto, a ingestão regular de álcool por $50 \%$ dos participantes é um aspecto que merece atenção, especialmente na orientação de que essa ingestão não ocorra antes do canto.

Os resultados relacionados ao tabagismo apontaram para maior conhecimento que esse grupo possui sobre os malefícios do tabaco, uma vez que apenas $4,5 \%$ dos participantes são tabagistas, e $22 \%$ abandonaram o uso do cigarro. Goulart et al. (2010) ${ }^{13}$, referiram que, no Brasil, a prevalência do tabagismo caiu de $35 \%$ para $18 \%$, no período de 1989 a 2003, sendo que, em 2006, essa taxa teve uma redução maior, chegando a $16 \%$. Programas de Saúde Pública, bem como as campanhas Nacionais da Voz são ações que incentivam o abandono do tabagismo, e o Ministério da Saúde e o Instituto Nacional de Câncer (INCA), assumiram o papel de organizar o Programa Nacional de Controle do Tabagismo.

Os resultados observados nesse estudo sugerem que o canto em coral desempenhou um papel importante na autopercepção da voz e possivelmente também na prevenção de alterações vocais. 


\section{CONCLUSÃO}

Com base nos dados obtidos pode-se concluir que os coristas avaliados apresentam um nível elevado de autopercepção de seus aspectos vocais, podendo sugerir que o canto coral desempenha um papel importante na promoção do bem estar vocal.

\section{AGRADECIMENTOS}

À Tatiane Camargo e Daniela Barbosa por ceder os dados coletados em sua pesquisa para análise.

À Professora Doutora Léslie pelo incentivo na publicação desse artigo.

\begin{abstract}
Purpose: to identify the vocal self-perception level of the vocal aspects, on the singers of a professional chorus. Method: participated 44 choristers, with ages between 20 and 75 years ( $\overline{\mathrm{X}} 51.5 \pm 14.36$ ) of both genders. All answered a questionnaire with 30 objective questions about complaints and selfperception of singing and speaking voice; experience in singing and vocal habits related to vocal health. Results: all the choristers self-defined your voices with positive characteristics, no statistically significant difference between spoken and singing voice. The choristers identified characteristics negatives to spoken voice and to singing voice, with statistical difference. Vocal complaints rate was $31 \%$ and $25 \%$ for spoken voice and singing voice respectively, but no statistical difference. $43 \%$ of participants were singing classes with an average time of 1.95 years $( \pm 2.29$ years) and the time of participation in chorus was 17.74 years ( \pm 18.45 years). About the habits, the average daily water intake was 1.7 liters $( \pm 0.92$ liters); $50 \%$ of the choristers drinks alcohol regularly; the numeric index of smokers was $4.5 \%$ and $22 \%$ of the choristers reported to have abandoned the use of cigarettes. There were no statistical correlation between smoking, drinking and singing experience with vocal complaints. Conclusion: conclude that the choristers analyzed have good level of self-perception of their vocal aspects, which suggests that chorus singing have importance in promotion of vocal health.
\end{abstract}

KEYWORDS: Voice; Aging; Habits; Self Concept

\section{REFERÊNCIAS}

1. Pereira E, Vasconselos M. O processo de Socialização no canto coral: Um estudo sobre as dimensões pessoal, interpessoal e comunitária. Música Hodie. 2007;7(1):99-120.

2. Hazlett D, Ball MJ. An acoustic analysis of the effects of ageing on the trained singer's voice. Logopedics Phoniatrics Vocology. 1996; 21(2):101-7.

3. Brown WS, Morris RJ, Michel JF. Vocal jitter and fundamental frequency characteristics in aged, female professional singers. J. Voice. 1990; 4(2):135-41.

4. Fuchs M, Meuret S, Thiel S, Täschner R, Dietz A, Gelbrich G. Influence of singing activity, age, and sex on voice performance parameters, on subjects' perception and use of their voice in childhood and adolescence. J. Voice. 2009;23(2):182-9.

5. Bicalho $A D$, Behlau $M$, Oliveira $O$. Termos descritivos da própria voz: comparação entre respostas apresentadas por fonoaudiólogos e não-fonoaudiólogos. Rev. CEFAC. 2010;12(4):543-50.

6. Kasama ST, Brasolotto AG. Percepção vocal e qualidade de vida. R. Pró-Fono Atual. Cient. 2007;19(1):19-28.

7. Camargo TF, Barbosa DA, Teles LCS. Características da fonetografia em coristas de diferentes classificações vocais. Rev. Soc. Bras. de Fonoaudiologia. 2007;12(1):10-7.

8. Amato RF. Ocanto coral como prática sócio-cultural e educativo-musica. Opus. 2007;13(1):75-96.

9. Machado MAMP, Aldrighi JM, Ferreira LP. Os sentidos atribuídos à voz por mulheres após a menopausa. Rev. Saúde Pública. 2005;2(39):261-9.

10. Menezes LN, Vicente LCC. Envelhecimento vocal em idosos instucionalizados. Rev. CEFAC. 2007;9(1):90-8.

11. Cerceau JSB, Alves CFT, GAMA ACC. Análise acústica da voz de mulheres idosas. Rev. CEFAC. 2009;11(1):142-9. 
12. Kubota ML. Considerações sobre a hidratação das pregas vocais [monografia]. São Paulo: CEFAC - Centro de Especializações em Fonoaudiologia Clínica; 1997.
13. Goulart, Engroff P, Ely LS, Sgnaolin V, Santos EF, Terra NL, Carli GA. Tabagismo em idosos. Rev. Bras. Geriatr. Gerontol. 2010;13(2):313-20.

Recebido em: 28/06/2012

Aceito em: 15/04/2013

Endereço para correspondência:

Fernanda Salvatico de Aquino

Rua Inácio Taveira, 18

São Paulo - SP

CEP 04658-110

E-mail: fernandasaquino@ hotmail.com 